\title{
Epidemiological and Clinical Profile of Pediatric Inflammatory Multisystem Syndrome - Temporally Associated with SARS-CoV-2 (PIMS-TS) in Indian Children
}

\author{
K Dhanalakshmi, ${ }^{1}$ Aishwarya Venkataraman, ${ }^{1}$ S Balasubramanian, ${ }^{1}$ Manoj Madhusudan, ${ }^{1}$ Sumanth \\ Amperayani, ${ }^{1}$ Sulochana Putilibai, ${ }^{2}$ Kalaimaran Sadasivam, ${ }^{3}$ Bala Ramachandran ${ }^{3}$ and AV Ramanan ${ }^{4}$ \\ From ${ }^{1}$ Departments of ${ }^{1}$ Paediatrics, ${ }^{2}$ Microbiology and ${ }^{3}$ Paediatric Intensive Care Unit, Kanchi Kamakoti CHILDS Trust Hospital, \\ Chennai, Tamil Nadu, India; and ${ }^{4}$ Bristol Royal Hospital for Children, Bristol, United Kingdom. \\ Correspondence to: Dr Aishwarya Venkataraman, Department of Paediatrics, Kanchi Kamakoti CHILDS Trust Hospital, $12 \mathrm{~A}$ \\ Nageswara Road, Nungambakkam, Chennai 600 034, India.draishwaryav@gmail.com
}

Background: We describe the demographic, clinical and laboratory findings along with the treatment and outcomes among children meeting the case definition of Pediatric Inflammatory Multisystem Syndrome - Temporally associated with SARS-CoV2 (PIMS-TS).

Methods: We analyzed the clinical and laboratory findings of children who presented with PIMS-TS during an 8-week period from May 4, 2020 to July 8, 2020.

Results: We report 19 children with a median age of 6 year (IQR: 13 months-16 years), who met the case definition of PIMS-TS. All of them presented with fever. Multi organ involvement $(79 \%)$, mucocutaneous involvement $(74 \%)$, cardiovascular symptoms
(63\%) and gastrointestinal symptoms (42\%) were the other features. Elevated levels of C-reactive protein was found in all of them and the majority of them had evidence of coagulopathy; intensive care admissions were needed in $12(63 \%)$ and vasoactive medications were given to $6(31.5 \%)$ children. There were no deaths.

Conclusion: Children with PIMS-TS present with a wide range of signs and symptoms. Fewer children in this series had coronary artery abnormalities, and there was a low incidence of RT-PCR positivity with high presence of SARS-CoV-2 antibodies.

Keywords: COVID-19, Hyper-inflammatory syndrome, Kawasaki disease, MIS-C, Toxic shock syndrome.

Published online: August 06, 2020; PII: S097475591600220 he impact of the coronavirus disease 2019 (COVID-19), caused by severe acute respiratory syndrome coronavirus 2 (SARSCoV-2) has been widespread. Initial reports worldwide showed that most children are asymptomatic or have mild or moderate disease [1-3]. However, there are now several reports of the pediatric multisystem inflammatory synd-rome associated with COVID-19 (PIMS-TS) in children globally [4-11].

In early May, the first published report of PIMS-TS or multisystem inflammatory syndrome in children associated with COVID-19 (MIS-C) was reported from India from our center. [11]. We hereby describe the demographic, clinical and laboratory findings of a series of cases of PIMS-TS seen since then in our center, so as to provide Indian data related to this syndrome.

\section{METHODS}

This study was conducted at a tertiary care children's hospital in Chennai, India. We analyzed children presenting to our hospital from May 4, 2020 to July 8, 2020 ( 8 week period), who satisfied the case definition of
PIMS-TS as defined by Royal College of Paediatrics and Child Health (RCPCH) [12]. Retrospectively, four children admitted during the month of April, 2020 were also included, as they met the criteria specified by RCPCH PIMS-TS definition. Data on the following parameters were collected: demographics, clinical findings, radiological findings, underlying comorbidities, echocardiographic findings, laboratory investigations, treatment received including intensive care interventions and outcome. This data is a part of a larger COVID-19 study in children presently undergoing at our institution, and was approved by the ethics committee. All children were included in the study after written informed consent of the caretaker.

Confirmed COVID-19 was defined as either positive SARS-CoV-2 real-time reverse-transcriptase polymerase chain reaction (RT-PCR) performed by Indian Council of Medical Research (ICMR) approved laboratories or positive antibody test performed with ICMR-approved YHLO SARS-CoV-2 IgG and IgM antibody titer assay kits (Shenzhen YHLO Biotech Co. Ltd.) as per manufacturer's instructions. 
Designated doctors and the study nurse collected all details in standardized and approved case report forms, which were then entered into to the Microsoft Excel spreadsheet. Vital signs (tachycardia, tachypnea and hypotension) were classified according to normal values for the age [13]. Data on various laboratory markers were collected and elevated levels were defined in relation to the normal levels for the age $[14,15]$. Cardiovascular involvement was described as children needing any of the following: fluid bolus $(>20 \mathrm{~mL} / \mathrm{kg}$ ) with or without vasoactive medications, an echocardiogram showing decreased left ventricular function $(\mathrm{EF}<55 \%)$, coronary artery abnormality, pericarditis or pericardial effusion, electrocardiogram (ECG) evidence of arrhythmias with or without elevated levels of troponin or pro BNP.

Statistical analyses: Data are presented as median (IQR), numbers and proportions. Statistical analyses were performed using SPSS version 24.0.

\section{RESULTS}

A total of 19 children with a median (IQR) age of 6 years (13 months-16 years) who met the criteria of PIMS-TS were included in this series. Between May 1 and July 8, 2020, 15 children were identified and four children were identified in April, 2020.

The male to female ratio was $1: 1.4$ and 9 children (47\%) were younger than 6 year. Of the 19 children, 15 (79\%) were tested for COVID 19 by RT-PCR and serological assays and $11(58 \%)$ were identified as confirmed cases of COVID-19. SARS-CoV-2 was confirmed by RT-PCR alone in three children (16\%), one child $(6 \%)$ had evidence from both RT-PCR and serological assay, 7 children (47\%) had positive serological assay alone whereas RT-PCR and serological assay was negative in 4 (27\%) children (Table I).

RT-PCR and/or serological assay negative or COVID19 status unknown children were included as they met the criteria as specified by RCPCH PIMS-TS definition. All children $(100 \%)$ presented with fever of more than 3 days and six (31\%) presented with lymphadenopathy. Multiorgan involvement was seen in majority of the children $(15 / 19,79 \%)$. Cardiovascular symptoms were reported in $12(63 \%)$ children, of which three had coronary artery abnormality at presentation (Table I).

Elevated CRP (median (IQR): 118 (73-298) mg/L) was noted in all 19 children (100\%). Coagulation parameters (PT, APTT and INR) were abnormal in 11/15 children (73\%) and D-dimer (median (IQR): 4,250 (339 $7328) \mathrm{ng} / \mathrm{mL}$ FEU) was elevated in $13 / 14$ (92.8\%) children (Table II).

INDIAN PEDIATRICS
Chest radiography was performed in 15 children, of which 5 showed evidence of lobar consolidation (unilateral). Ultrasound scan of abdomen was performed in 5 children of whom one was suggestive of as possible appendicitis. CT chest and abdomen was performed in the same child, which showed evidence of right lower lobe consolidation. Coronary artery abnormality (dilatation without aneurysm, $Z$ score $<2.5$ ) was seen in three children with one of them having evidence of minimal pericardial effusion.

Of the 19 children, 5 (26\%) received intravenous immunoglobulins (IVIG) alone, whereas three children $(16 \%)$ were treated with steroids alone; 8 children $(42 \%)$ received both IVIG and steroids, and one child received IVIG and tocilizumab. Aspirin was given in 16 (84.2\%) children and two children were not given any immunomodulatory agents. All 19 children received broadspectrum antibiotics at presentation, which were discontinued after negative culture results. No organisms were isolated from blood cultures.

Only one child had underlying co-morbidity (global developmental delay) and one child presented with features mimicking appendicitis along with positive SARS-CoV-2 antibodies. Median length of hospitalization was 6 days (IQR 3-13 days) and 12 (63\%) children required PICU support. There was no mortality in our series.

\section{DISCUSSION}

This study is the first series from India describing children presenting with PIMS-TS. Consistent with published data from Europe and US, children in this study also presented with signs and symptoms mimicking complete or incomplete Kawasaki disease (KD), toxic shock syndrome (TSS), hemophagocytic lymphohistiocytosis (HLH) and/ or macrophage activation syndrome (MAS) $[4,5,10]$. Although, cardiac dysfunction is the most commonly reported organ dysfunction $[5,7,12]$, a notable finding in our series was that a fewer number of children were identified to have echocardiographic evidence of coronary artery changes $(3 / 19,16 \%)$, though a significant number of children $(57 \%)$ developed hypotension requiring admission to the PICU for vasoactive medications. Likewise, when compared to the available data, fewer children (42\%) in our series presented with gastrointestinal symptoms as against up to $80 \%$ in literature $[4-6,16]$, and more than two-third $(74 \%)$ presented with mucocutaneous manifestations.

Clinical presentation, epidemiology and pathogenesis of PIMS-TS are still unclear and evolving, but cases of PIMS-TS seem to appear few weeks after the COVID-19 peak in the population $[5,17,18]$. The COVID-19 peak in 
Table I Demographic and Clinical Characteristics of Children With PIMS-TS $(N=19)$

\begin{tabular}{|c|c|c|c|}
\hline & \multirow[t]{2}{*}{ All children } & \multicolumn{2}{|c|}{${ }^{\wedge} R T-P C R /$ Serology } \\
\hline & & Positive $(n=11)$ & Negative/unknown $(n=8)$ \\
\hline Age, median (range) & 6 y (1y 1m-16y 9m) & 8.2 y $(2$ y $10 \mathrm{~m}-16$ yr $9 \mathrm{~m})$ & 4.2 y $(1 \mathrm{yr} 1 \mathrm{~m}-11 \mathrm{y} 1 \mathrm{~m})$ \\
\hline Male & $8(42)$ & $4(36)$ & $4(50)$ \\
\hline Comorbidity & $1 / 19(5.2)$ & $1 / 11(9)$ & 0 \\
\hline RT-PCR positive & $4 / 15$ & $4 / 11$ & 4 negative, 4 not tested \\
\hline Serology positive & $8 / 15$ & $8 / 11$ & 4 negative, 4 not tested \\
\hline Fever & $19(100)$ & $11(100)$ & $8(100)$ \\
\hline Lymphadenopathy & $6(31.5)$ & $1(9)$ & $5(62.5)$ \\
\hline GI symptoms & $8(42)$ & $6(54.5)$ & $2(25)$ \\
\hline Abdominal pain & 8 & 6 & 2 \\
\hline Vomiting & 6 & 4 & 2 \\
\hline Diarrhea & 3 & 3 & 0 \\
\hline Mucocutaneous & $14(74)$ & $6(54.5)$ & $8(100)$ \\
\hline Rash & 12 & 4 & 8 \\
\hline Edema & 10 & 4 & 6 \\
\hline Congested conjunctiva & 9 & 3 & 6 \\
\hline Oral mucosa involved & 9 & 2 & 7 \\
\hline CVS symptoms & $12(63)$ & $9(81.8)$ & $3(37.5)$ \\
\hline Hypotension & 10 & 9 & 1 \\
\hline Acute kidney injury & $3(16)$ & $3(27.2)$ & 0 \\
\hline Respiratory symptoms & $8(42)$ & $5(45.4)$ & $3(37.5)$ \\
\hline Neurological symptoms & $6(31)$ & $3(27.2)$ & $3(37.5)$ \\
\hline Meeting KD criteria & $7(36.8)$ & $1(9)$ & $6(75)$ \\
\hline PICU admission & $12(63)$ & $10(91)$ & $2(25)$ \\
\hline Mechanical ventilation & 0 & 0 & 0 \\
\hline HHFNC & 1 & 1 & 0 \\
\hline Nasal cannula oxygen & 4 & 4 & 0 \\
\hline Fluid bolus (>20 mL/kg) & $10(52.6)$ & $9(81.8)$ & $1(12.5)$ \\
\hline Vasoactive support & $6(31.5)$ & $6(54.5)$ & 0 \\
\hline IVIG used & $15(79)$ & $7(63.6)$ & $8(100)$ \\
\hline Steroids used & $11(58)$ & $8(72.7)$ & $3(37.5)$ \\
\hline Tocilizumab ( $8 \mathrm{mg} / \mathrm{kg}$ ) used & $1(5.2)$ & $1(9)$ & 0 \\
\hline Aspirin used & $16(84.2)$ & $8(72.7)$ & $8(100)$ \\
\hline
\end{tabular}

PIMS-TS: Pediatric Inflammatory Multisystem Syndrome: Temporally Associated with SARS-CoV-2; All values in no. (\%); GI: gastrointestinal, PICU: Pediatric intensive care unit; HHFNC: High flow nasal cannula oxygen; IVIG: Intravenous immunoglobulin; CVS: Cardiovascular system; RT-PCR: Reverse transcriptase polymerase chain reaction; ${ }^{\wedge} R T-P C R$ results available in only 15 children - $4 / 11$ were positive in first group, and in second group 4/8 were negative and results were unknown in remaining 4.

the community is possibly yet to occur in several cities in India, and we postulate that we may also see a significant increase of PIMS-TS among children in the coming days.

A positive serologic assay for SARS-CoV-2 or RT-PCR has been a consistent finding in the literature [7,8]; although, there have also been published reports with negative results for SARS-CoV-2 [10]. Most of the children in this study (58\%) had laboratory confirmed SARS-CoV2 infection. Serology testing or RT-PCR could not be performed in four children as they presented to us at the beginning of COVID-19 pandemic in Chennai. These four children had no microbiological evidence for other infections. They had multi-organ dysfunction with elevated inflammatory makers (CRP, D-dimer and ESR) in addition to neutrophilia and lympho-penia. However, we plan to perform serological assay in these children during their follow up to establish a link between their symptoms and SARS-CoV-2. PIMS-TS generally tend to occur in older children (reported median age 8 years) $[5,6,10]$, which is 
Table II Profile of Laboratory Markers in Children With PIMS-TS ( $N=19)$

\begin{tabular}{|c|c|c|c|}
\hline & \multirow[t]{2}{*}{ All children } & \multicolumn{2}{|c|}{${ }^{\wedge} R T-P C R /$ Serology } \\
\hline & & Positive $(n=11)$ & Negative/unknown $(n=8)$ \\
\hline Elevated CRP & $19(100)$ & $11(100)$ & $8(100)$ \\
\hline Elevated troponin $(\mathrm{pg} / \mathrm{mL})$ & $1 / 6(16.6)$ & $1 / 5$ & $0 / 1$ \\
\hline Elevated NT pro BNP (pg/mL) & $3 / 4(75)$ & $3 / 3(100)$ & $0 / 1$ \\
\hline${ }^{\&}$ Elevated fibrinogen & $7 / 9(77.7)$ & $6 / 7(85.7)$ & $1 / 2(50)$ \\
\hline Elevated D-dimer (ng/mL FEU) & $13 / 14(92.8)$ & $10 / 11(91)$ & $2 / 3(67)$ \\
\hline \%Hypoalbuminemia (g/dL) & $11 / 18(61.1)$ & $7 / 11(63.6)$ & $4 / 7(57.1)$ \\
\hline${ }^{\circledR}$ Hyponatremia (mmol/L) & $11 / 19(58)$ & $7 / 11(63.6)$ & $5 / 8(63)$ \\
\hline Elevated LDH (U/L) & $7 / 13(53.8)$ & $4 / 10(40)$ & $3 / 3(100)$ \\
\hline Neutrophilia $\left(\right.$ per $\mathrm{m}^{3}$ ) & $13(68.4)$ & $6(54.5)$ & $7(87.5)$ \\
\hline Lymphopenia $\left(\right.$ per $\mathrm{m}^{3}$ ) & $7(36.8)$ & $6(54.5)$ & $1(12.5)$ \\
\hline${ }^{\wedge}$ High ferritin $(\mathrm{ng} / \mathrm{mL})$ & $3(21.4)$ & $3 / 10(30)$ & $0 / 4$ \\
\hline Anemia $(\mathrm{mg} \%)$ & $6(31.5)$ & $5(45.4)$ & $1(12.5)$ \\
\hline Thrombocytopenia (per $\mathrm{mm}^{3}$ ) & $3(15.7)$ & $3(27.2)$ & 0 \\
\hline${ }^{\#}$ Elevated ESR $(\mathrm{mm} / \mathrm{h})$ & $9 / 11(81.8)$ & $4 / 5(80)$ & $5 / 6(83.3)$ \\
\hline Transaminitis (U/L) & $5(26.3)$ & $4(36.3)$ & $1(12.5)$ \\
\hline Deranged coagulation & $11 / 15(73.3)$ & $9 / 11(81.8)$ & $2 / 4(50)$ \\
\hline Abnormal chest $X$-ray & $5 / 15(33.3)$ & $5 / 11(45.4)$ & $0 / 4$ \\
\hline Coronary artery changes* & 3 & $1^{¥}$ & 2 \\
\hline Three systems involved & $7(36.8)$ & $4(36.3)$ & $3(37.5)$ \\
\hline Four systems involved & $6(31.5)$ & $5(45.5)$ & $1(12.5)$ \\
\hline
\end{tabular}

PIMS-TS: Paediatric Inflammatory Multisystem Syndrome: Temporally Associated with SARS-CoV-2; \#median (IQR) ESR: 86 (15-140) mm/h; Amedian (IQR) ferritin 238 (220-1230) ng/mL; * median (IQR) LDH: 451 (307-751) U/L; @ median (IQR) hyponatremia: 132 (130-139) mmol/L; \%median (IQR) hypalbumenia: 3 (2.3-3.4)g/dL; ${ }^{\star}$ Median (IQR) Fibrinogen: 458 (228-669) mg/dL; PICU: Pediatric intensive care unit; CRP: Creactive protein (>30 mg/l); Troponin: T (>4pg/mL); NT pro BNP: N Terminal PRO B Type Natriuretic Peptide (>180 pg/mL FEU), Fibrinogen (>400 $\mathrm{mg} / \mathrm{dL})$, D-dimer ( $>500 \mathrm{ng} / \mathrm{mL} \mathrm{FEU})$, Hypoalbuminemia $(<3.5 \mathrm{~g} / \mathrm{dL})$, Hyponatremia $(<135 \mathrm{mmol} / \mathrm{l}), \mathrm{LDH}(>460 \mathrm{U} / \mathrm{l})$, Neutrophilia $\left(>7700 / \mathrm{mm}^{3}\right)$, Lymphopenia $\left(<1500 / \mathrm{mm}^{3}\right)$, Anemia $(<9 \mathrm{mg} \%)$, Thrombocytopenia $\left(<1.5 \mathrm{l} / \mathrm{mm}^{3}\right)$, Ferritin $(>500 \mathrm{ng} / \mathrm{mL})$, ESR-Erythrocyte Sedimentation Rate $(>40$ $\mathrm{mm} / \mathrm{hr}$ ), Transaminitis- (Alanine amino transferase (ALT)/Aspartate amino transferase (AST) >40IU/l), PT: Prothrombin time; INR: International Normalized Ration $>1.2 ;{ }^{*}$ Coronary artery changes-dilatation without aneurysms ( $z$ score $<2.5$ ); ${ }^{*}$ Evidence of minimal pericardial effusion in addition to coronary artery dilatation.

slightly more than that seen in our patients. Laboratory testing in our group generally showed significant elevation of inflammatory markers, as reported earlier $[6,19]$.

Currently there is no consensus regarding management of children with PIMS-TS; although there has been a recently published review suggesting a treatment flowchart [20]. IVIG $(2 \mathrm{~g} / \mathrm{kg})$ has been most commonly used as first line therapy with many children receiving additional highdose steroids $[5-7,16]$. Nearly half of the children $(42 \%)$ in this series received both IVIG and steroids, with a few children requiring a second dose of IVIG and one child needing additional immuno-modulatory medication. The role of aspirin in children with hyperinflammation without $\mathrm{KD}$ is not yet described, though it has been used by many in PIMS-TS [10,11]. Lack of uniform guideline for management reinforces the fact that further studies are required to establish optimal treatment in PIMS-TS.

The main limitations of the study are relatively smaller number of patients and a shorter duration of study; hence, we are unable to provide data on long-term sequelae of PIMS-TS. Another limitation is absence of serological confirmation of SARS-CoV-2 infection in nearly one-fifth of the children.

Our study is one of the first series from Asia describing PIMS-TS in children. We report fewer coronary artery abnormalities, as compared to the existing data on PIMSTS. Finally, we also report low incidence of RT-PCR positivity with increased presence SARS-CoV-2 antibodies. This study underscores the occurrence of PIMS-TS in children in India and will increase awareness of the disease among the clinicians, so as to enable early recognition and prompt management.

Ethics approval: CTMRF-KKCTH Ethics committee; No. ECR/ 676/Inst/TN/2014/RR-17, dated June 2, 2020.

Acknowledgements: Dr Anand Manoharan, Director Clinical research, CTMRF and the following Senior Pediatric 


\section{WHAT THIS STUDY ADDS?}

- Lower age and lesser echocardiographic abnormalities were observed among children with PIMS-TS.

- Prompt recognition and treatment with immunomodulatory agents are likely to result in favorable outcome in PIMS-TS.

Consultants at Kanchi Kamakoti CHILDS Trust Hospital (KKCTH): Dr Janani Sankar, Dr T Vasanthi, Dr T Ravikumar, Dr S Shivabalan, Dr Asokan and Dr Rajkumar for their support and contribution.

Contributors: SBS, AVR: concept and design; KD, MM, AV: acquisition, analysis and interpretation of data; AV, SBS, SA, KS: drafting of the manuscript; MM,AV: statistical analysis; AV, SP: analysis of laboratory assays; KD, AV, SBS, SA, SP, KS, BR, AVR, SA: critical revision of the manuscript for important intellectual content; KD, AV, SBS, SA, MM, SP, KS, BR, AVR: final approval of the version to be published.

Funding; Child Trust Medical Research Foundation (CTMRF); Competing interests: None stated.

\section{REFERENCES}

1. Castagnoli R, Votto M, Licari A, et al. Severe acute respiratory syndrome corona-virus 2 (SARS-CoV-2) infection in children and adole-scents: A systematic review. JAMA Pediatr. 2020;174:882-9.

2. Meena J, Yadav J, Saini L, Yadav A, Kumar J. Clinical features and outcome of SARS-CoV-2 infection in children: A systematic review and meta-analysis. Indian Pediatr. 2020;57:820-6.

3. Dong Y, Mo X, Hu Y, et al. Epidemiology of COVID-19 among children in China. Pediatrics. 2020;145: e20200702.

4. Dufort EM, Koumans EH, Chow EJ, et al. Multisystem inflammatory syndrome in children in New York state. N Engl J Med. 2020; 383:347-58.

5. Feldstein LR, Rose EB, Horwitz SM, et al. Multisystem inflammatory syndrome in US children and adolescents. $\mathrm{N}$ Engl J Med. 2020;383: 334-46.

6. Whittaker E, Bamford A, Kenny J, et al. Clinical characteristics of 58 children with a pediatric inflammatory multisystem syndrome temporally associated with SARSCoV-2. [published online ahead of print, 2020 Jun 8]. JAMA. 2020; 2010369.

7. Verdoni L, Mazza A, Gervasoni A, et al. An outbreak of severe Kawasaki-like disease at the Italian epicentre of the SARS-CoV-2 epidemic: An observational cohort study. Lancet. 2020; 395:1771-8.

8. Cabrero-Hernández M, García-Salido A, Leoz-Gordillo I, et al. Severe SARS-CoV-2 infection in children with suspected acute abdomen: A case series from a tertiary hospital in Spain. Pediatr Infect Dis J. 2020;39:e195-8.

9. Zahra B, Mathilde M, Fanny B, et al. Acute heart failure in multisystem inflammatory syndrome in children (MIS-C) in the context of global SARS-CoV-2 pandemic [published online ahead of print, 2020May 17]. Circulation. 2020;10.1161/
CIRCULATIONAHA. 120.048360.

10. Riphagen S, Gomez X, Gonzalez-Martinez C, Wilkinson $\mathrm{N}$, Theocharis P. Hyperinflammatory shock in children during COVID-19 pandemic. Lancet. 2020;395:1607-8.

11. Balasubramanian S, Nagendran TM, Ramachandran B, Ramanan AV. Hyper-inflammatory syndrome in a child with covid-19 treated successfully with intravenous immuno-globulin and tocilizumab. Indian Pediatr. 2020;57: 681-3.

12. The Royal College of Paediatrics and Child Health. Paediatric multisystem inflammatory syndrome temporally associated with COVID-19; Available from: https://www. rcpch.ac.uk/sites/default/files/2020-05/COVID-19-Paediatric-multisystem-inflammatory syndrome-20200501. pdf. Accessed August 5, 2020.

13. Tschudy MM, Arcara KM, editors. The Harriet Lane handbook: A Manual for Pediatric House Officers. Philadelphia, PA: Elsevier Mosby; 2012.

14. Bain BJ, Bates I, Laffan MA, Lewis SM, editors. Dacie and Lewis Practical Haematology. Pathology, 12th edition. Philadelphia, PA; Elsevier: 2016.

15. Lopez J, Burtis CA, Ashwood ER, Bruns DE, editors. Tietz Textbook of Clinical Chemistry and Molecular Diagnosis. Philadelphia, PA; Elsevier.

16. Davies P, Evans C, Kanthimathinathan HK, et al. Intensive care admissions of children with paediatric inflammatory multisystem synd-rome temporally associated with SARSCoV-2 (PIMS-TS) in the UK/: A multicentre observational study [published online ahead of print, $2020 \mathrm{Jul}$ 9] Lancet Child Adolesc Health. 2020;S2352-4642(20)30215-7. [published correction in Lancet Child Adolesc Health. $2020 \mathrm{Jul} 17]$.

17. Rowley AH. Multisystem inflammatory syndrome in children and Kawasaki disease: Two different illnesses with overlapping clinical features Pediatr. 2020;224:129-32.

18. Nakra NA, Blumberg DA, Herrera-Guerra A, Lakshminrusimha S. Multi-system inflammatory syndrome in children (MIS-C) following SARS-CoV-2 infection: Review of clinical presentation, hypothetical pathogenesis, and proposed management. Children (Basel). 2020;7:E69. Published 2020 Jul 1.

19. $\mathrm{Wu} \mathrm{H}, \mathrm{Zhu} \mathrm{H}$, Yuan $\mathrm{C}$, et al. Clinical and immune features of hospitalized pediatric patients with coronavirus disease 2019 (COVID-19) in Wuhan, China. JAMA Network Open. 2020;3:e2010895.

20. Bhat CS, Gupta L, Balasubramanian S, Singh S, Ramanan AV. Hyperinflammatory syndrome in children associated with COVID-19: Need for awareness. Indian Pediatr. 2020;57:929-35. 